\title{
FGFR3 silencing by siRNA inhibits invasion of A549 cells
}

\author{
YUHUA LI* ${ }^{*}$ XIGUANG LIU*, HONGJUN ZHANG, TAO JIANG, \\ WENJING XIAO, SHUFEN ZHAO, XIAOYUN YU and FANJIE HAN
}

Department of Oncology, The Affiliated Hospital of Qingdao University, Qingdao, Shandong 266003, P.R. China

Received March 7, 2015; Accepted February 11, 2016

DOI: $10.3892 / 01.2016 .5278$

\begin{abstract}
The present study identified that fibroblast growth factor receptor 3 (FGFR3) was significantly upregulated in bone metastasis of lung adenocarcinoma. RNA interference (RNAi) is a powerful approach for treating a wide range of human diseases, including cancer, through downregulating the expression of selected genes. In the present study, the invasiveness of A549 cells cultured in vitro was altered by small interfering (si)RNA targeting FGFR3, and the regulatory effect of silencing FGFR3 on the expression levels of E-cadherin and matrix metalloproteinase (MMP) 9 was investigated. Human lung adenocarcinoma A549 cells were transfected with synthetic specific siRNAs targeting a fragment of the FGFR3 gene (namely, siRNA-855, siRNA-1447 and siRNA-2076) or with negative control (NC) siRNA. Cells were divided into five groups (A, siRNA-855 group; B, siRNA-1447 group; C, siRNA-2076 group; D, NC-siRNA group; and E, blank control group). The effect of the above siRNAs targeting FGFR3 on the invasion capacity of A549 cells was detected by Transwell assay. siRNAs against FGFR3 were transfected into A549 cells with by Lipofectamine ${ }^{\circledR} 2000$, and the expression levels of FGFR3, E-cadherin and MMP9 were measured by reverse transcription-quantitative polymerase chain reaction and western blot assay. The experimental findings indicated that the expression levels of FGFR3 and MMP9 were significantly reduced in the siRNA-FGFR3-transfected groups (A-C groups), compared with those in the D and $\mathrm{E}$ groups $(\mathrm{P}<0.01)$. In addition, the expression levels of $\mathrm{E}$-cadherin were markedly elevated in the A-C groups, compared with those in the $\mathrm{D}$ and $\mathrm{E}$ groups $(\mathrm{P}<0.01)$. There was no significant difference in E-cadherin expression between the A-C groups, or between the $\mathrm{D}$ and $\mathrm{E}$ groups $(\mathrm{P}>0.05)$. These results indicated that siRNA-FGFR3 was able to decrease the invasiveness of
\end{abstract}

Correspondence to: Professor Xiguang Liu, Department of Oncology, The Affiliated Hospital of Qingdao University, 16 Jiangsu Road, Qingdao, Shandong 266003, P.R. China

E-mail: yihehandsome@126.com

*Contributed equally

Key words: FGFR3, siRNA, RNAi, invasion, migration, reverse transcription-quantitative polymerase chain reaction
A549 cells, inhibit the expression of MMP9 and increase the expression of E-cadherin by downregulating the expression of FGFR3. Taken together, the results of the present study indicated that the upregulation of E-cadherin expression and the downregulation of MMP9 expression are able to inhibit the migration of A549 cells, and siRNA silencing FGFR3 acts as a tumor suppressor in these cells.

\section{Introduction}

Non-small cell lung cancer (NSCLC) is the most common type of lung cancer and the leading cause of cancer-associated mortality worldwide, which mostly results from local invasion or distant metastases, rather than being due to primary tumors (1). The 5-year survival rate of advanced lung cancer has been reported to be $<15 \%$ (2). It has been recognized that certain tumors present a predilection for metastasis to specific organs $(3,4)$, being bone, brain, liver and lung the most frequent target organs of metastasis (4). The mechanism of tumor development in lung cancer is likely to be associated with genetic diseases that occur when certain genes are mutated or exhibit abnormalities such as deletions (3). Novel strategies for the treatment of lung cancer require the identification of novel molecules that are able to inhibit the invasiveness and metastasis of lung cancer cells (5).

Small interfering (si)RNA molecules consist of 21-23 nucleotides in length and display a characteristic and highly specific structure containing 2-3 nucleotides in the $3^{\prime}$ overhangs and 5' phosphate and 3' hydroxyl groups to prevent erroneous gene silencing, in addition to a sense (passenger) strand and an antisense (guide) strand (6). The remarkable advances in biology and cancer therapy that had occurred during the past recent decades have led to the development of siRNA technology, which is an effective method to sequence-specific knockdown or reduce gene function through homology-dependent degradation of the corresponding target messenger (m)RNA, via a phenomenon known as RNA interference (RNAi), which is one of the most rapidly growing topics of research for the treatment of various diseases, including cancer $(6,7)$. Despite the caveats and ongoing challenges such as off-target effects and false discovery rates, RNAi remains a powerful approach for reverse genetics in large-scale functional analysis conducted in cultured cells and in various in vivo systems (6). Furthermore, the development of siRNA therapeutics is rapidly gaining momentum at present (6). 
Lung cancer is the leading cause of cancer-associated mortality and morbidity worldwide (8), and NSCLC is responsible for almost $80 \%$ of all lung cancer-associated mortalities, mostly resulting from tumor invasion and metastasis $(8,9)$. Fibroblast growth factor receptor 3 (FGFR3) is a member of the transmembrane receptor tyrosine kinase (RTK) family, which interacts with FGF ligands and participates in the regulation of cell proliferation, differentiation and tumorigenesis $(10,11)$. Previous studies demonstrated that the expression levels of FGFR3 were significantly upregulated in metastasis of NSCLC, compared with normal lung tissues, according to the results of quantitative analysis of FGFR3 expression in humans $(3,10,12)$. In addition, FGFR 3 has been demonstrated to be involved in the RAS/RAF/mitogen-activated protein kinase (MAPK) kinase (MEK)/MAPK signaling pathway through the activation of p90 ribosomal S6 kinase (13).

Migration and invasion are critical steps in the initial progression of cancer that facilitate metastasis $(14,15)$. In particular, the critical steps required for the initiation and progression of tumor invasion are epithelial-mesenchymal transition (EMT) and extracellular matrix (ECM) degradation (14). E-cadherin is an important marker of EMT (15), while matrix metalloproteinase (MMP)9 is a member of the MMP family of enzymes, which are involved in the degradation of the ECM $(16,17)$. Increased levels of MMP9 have been correlated with tumor aggressiveness in numerous types of cancer (18).

RNAi is a popular method for exploring gene function, which suppresses the expression of a specific gene of interest in transfected mammalian cell cultures (19). In the present study, siRNA-FGFR3 significantly inhibited the expression of FGFR3 by directly targeting FGFR3 in A549 cells. The present authors hypothesized that siRNA-FGFR3 was able to silence FGFR3 and inhibit the migration of A549 cells by decreasing the expression levels of E-caherin and MMP9 in these cells. To test the above hypothesis, cell invasion ability was assessed by Transwell invasion assay, reverse transcription-quantitative polymerase chain reaction (RT-qPCR) and western blot analysis in A549 cells transfected with siRNA-FGFR3.

\section{Materials and methods}

Cell line and culture. A549 lung cancer cells were obtained from the Shanghai Cell Bank Chinese Academy of Sciences (Shanghai, China). Cells were cultured in RPMI-1640 medium (Gibco; Thermo Fisher Scientific, Inc., Waltham, MA, USA) supplemented with $10 \%$ fetal bovine serum (Hyclone; GE Healthcare Life Sciences, Logan, UT, USA), $40,000 \mathrm{mU} / \mathrm{ml}$ penicillin (Mediatech, Inc.; Corning Life Sciences, Manassas, VA, USA) and $40 \mu \mathrm{g} / \mathrm{ml}$ streptomycin (Mediatech, Inc.; Corning Life Sciences), at $37^{\circ} \mathrm{C}$ in a humidified incubator with $5 \% \mathrm{CO}_{2}$. Upon reaching $90 \%$ confluence, the cells were dissociated using $0.25 \%$ trypsin (Hyclone, Shanghai, China), and subcultured.

siRNA transfection. Cells were transfected with siRNAs targeting FGFR3 or with control siRNA using Lipofectamine $^{\circledR} 2000$ (Lipo2000) (Invitrogen; Thermo Fisher
Scientific, Inc.). The transfection was performed at $37^{\circ} \mathrm{C}$ in a humidified incubator with $5 \% \mathrm{CO}_{2}$. The siRNAs were designed and synthesized by Shanghai GenePharma Co., Ltd. (Shanghai, China), and their sequences were queried with Basic Local Alignment Search Tool [National Center for Biotechnology Information (NCBI), Bethesda, MD, USA], and identified as FGFR3-specific sequence, once homology with other genes was excluded. In total, 3 groups of cells were transfected with siRNAs whose sequence was specific for FGFR3 (namely, siRNA-855, siRNA-1447 and siRNA-2076). Another group of cells was transfected with a fluorescein amidite (FAM)-labeled nonspecific siRNA, that served as negative control (NC)siRNA. The sequences of the siRNAs used in the present study were as follows: siRNA-855 sense, 5'-GCAUUGGAGGCAUCAAGCUTT-3' and anti-sense, 5'-AGCUUGAUGCCUCCAAUGCTT-3'; siRNA-1447 sense, 5'-GCUGAAAGACGAUGCCACUTT-3' and anti-sense, 5'-AGUGGCAUCGUCUUUCAGCTT-3'; siRNA-2076 sense, 5'-GCACACACGACCUGUACAUTT-3' and anti-sense, 5'-AUGUACAGGUCGUGUGUGCTT-3'; and NC-siRNA sense, 5'-UUCUCCGAACGUGUCACG UTT-3' and anti-sense, 5'-ACGUGACACGUUCGGAGA ATT-3'. When A549 cells seeded into 6-well plates reached $80 \%$ confluence, transfection was conducted by mixing $5 \mu 1$ siRNA with $5 \mu \mathrm{l}$ Lipo2000 in a final volume of $2,000 \mu \mathrm{l}$ RPMI-1640 medium (Gibco; Thermo Fisher Scientific, Inc.) containing $10 \%$ serum without antibiotics, according to the manufacturer's protocol. Cell morphology and transfection efficiency were evaluated at $6 \mathrm{~h}$ following transfection. Cells were divided into 5 groups termed A-E, and transfected with siRNA-855, siRNA-1447, siRNA-2076, NC-siRNA and blank control, respectively. Transfections were performed in triplicate and the experiment was repeated $\geq 3$ times.

Transwell invasion assay. Cell invasion was assessed by Transwell invasion assay using $8-\mu \mathrm{m}$ Matrigel-coated invasion chambers (Costar; Corning Life Sciences), according to the manufacturer's protocol. Briefly, A549 cells $\left(0.5 \times 10^{5}\right.$ cells/well $)$ were suspended in $200 \mu 1$ serum-free medium and seeded on the upper chamber of a 24-well Transwell migration chamber (Costar; Corning Life Sciences). The lower compartment was filled with $750 \mu$ l complete medium. Following 16 -h culture at $37^{\circ} \mathrm{C}$ in a humidified incubator with $5 \% \mathrm{CO}_{2}$, those cells that were retained on top of the membrane were removed with a cotton swab, while those cells that had migrated to the bottom side (invaded cells) were fixed with $10 \%$ formalin (Costar; Corning Life Sciences), stained with $0.1 \%$ crystal violet (Costar; Corning Life Sciences) and observed under a light microscope (Olympus BX41; Olympus Corporation, Tokyo, Japan) at 200x magnification. The number of penetrating cells present in 9 random fields was counted, and the experiment was repeated $\geq 3$ times.

$R T-q P C R$. Total RNA was isolated from A549 cells using TRIzol reagent (Invitrogen; Thermo Fisher Scientific, Inc.) at $36 \mathrm{~h}$ post-transfection, according to the manufacturer's protocol. The concentration and purity of the extracted RNA was quantified by its absorbance at $260 \mathrm{~nm}$ using a BioPhotometer (D30; Eppendorf, Hamburg, Germany). 

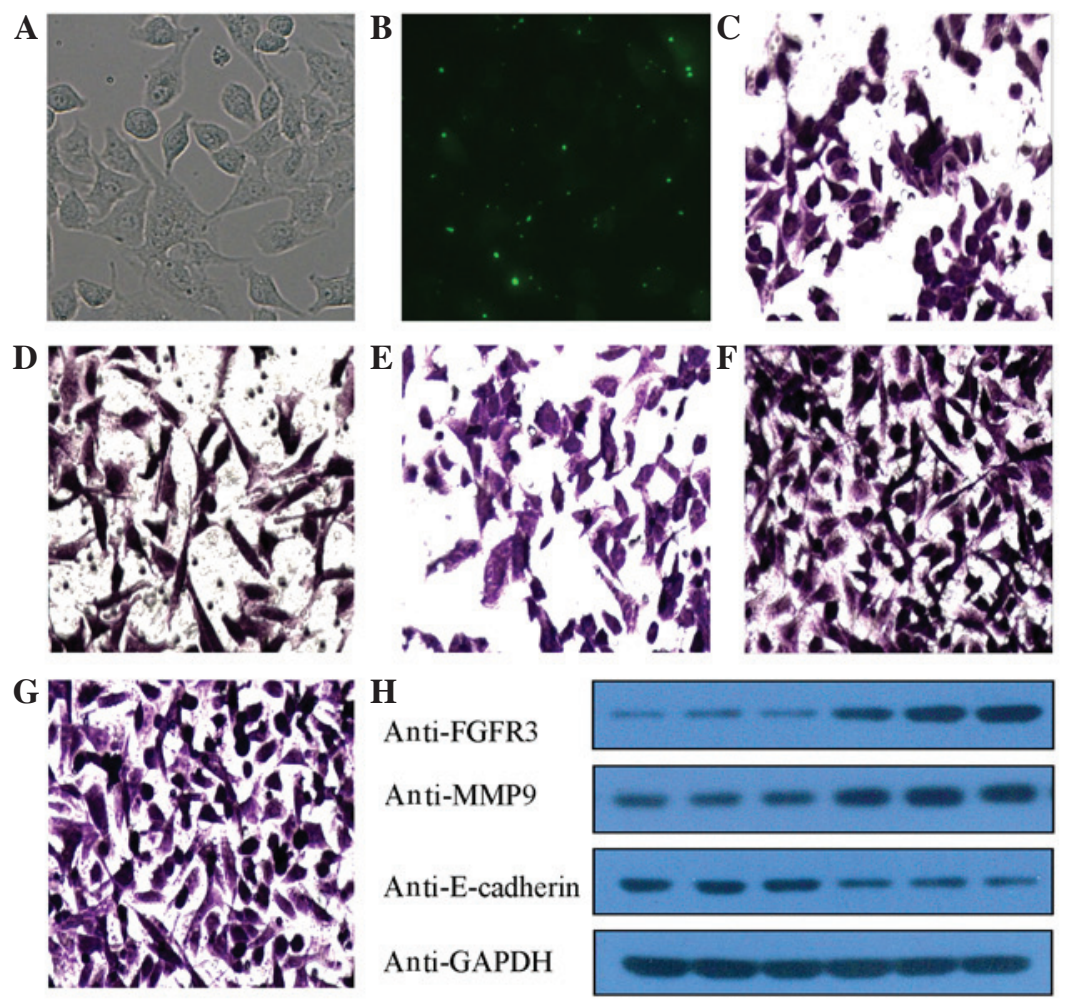

Figure 1. (A and B) Following transfection with small interfering RNA labeled with fluorescein amidite for $6 \mathrm{~h}$, green fluorescence emission produced by blue light excitation was observed in the transfected A549 cells under an inverted fluorescence microscope. The transfection efficiency was determined to be 70-80\%. (A) shows image under high power microscope (magnification, x200) and (B) shows fluorescence microscopy (magnification, x200). Following transfection for $6 \mathrm{~h}$, A549 cells in (C) group A, (D) group B, (E) group C, (F) group D and (G) group E were subjected to Transwell assay. Cells that migrated to the bottom chamber (invaded cells) were fixed with $10 \%$ formalin, stained with $0.1 \%$ crystal violet and observed under 200x magnification. (H) Compared with D and E groups, the protein expression levels of fibroblast growth factor receptor 3 and matrix metalloproteinase 9 were downregulated in groups A-C, whereas the expression of E-cadherin protein was upregulated in these groups.

RT-qPCR was conducted with SYBR Green II (catalogue number RR037A; Takara Bio, Inc., Otsu, Japan), according to the manufacturer's protocol. The experiment was repeated $\geq 3$ times. The PCR primers were designed and synthesized by Sangon Biotech Co., Ltd. (Shanghai China), and their sequences were as follows: FGFR3 sense, 5'-TCAGGG TGGTCTCTTCTTGG-3' and anti-sense, 5'-CGTCGCTGG GTTAACAAAT-3'; MMP9 sense, 5'-TTCCAAACCTTT GAGGGCGA-3' and anti-sense, 5'-GCAAAGGCGTCG TCAATCAC-3'; E-cadherin sense, 5'-GGGTTATTCCTC CCATCAGC-3' and anti-sense, 5'-GTCACCTTCAGCCAT CCTGT-3'; and glyceraldehyde-3-phosphate dehydrogenase (GAPDH) sense, 5'-GATCATCAGCAATGCCTCCTG-3' and anti-sense, 5'-GAGTCCTTCCACGATACCAAAG-3'. The sequence of the specific primers for FGFR3, MMP9, E-cadherin and GAPDH were based on the corresponding GenBank sequence (NCBI) (FGFR3 GeneID, NM_000142.4 GI:254028235; MMP9 GeneID, NM_004994.2 GI:74272286; E-cadherin GeneID, NM_004360.3 GI:169790842; and GAPDH GeneID, NM_001256799.1 GI:378404907). PCR was performed under the following conditions: Initial denaturation at $95^{\circ} \mathrm{C}$ for $5 \mathrm{~min}$, followed by 35 cycles of $95^{\circ} \mathrm{C}$ for $30 \mathrm{sec}, 55^{\circ} \mathrm{C}$ for $1 \mathrm{~min}$ and $72^{\circ} \mathrm{C}$ for $1 \mathrm{~min}$, with a final extension step at $72^{\circ} \mathrm{C}$ for $5 \mathrm{~min}$. GAPDH served as an internal control. To ensure the specificity of each set of primers, the melting point temperatures of the amplicons generated by PCR were evaluated using High Resolution Melt Software, a first-derivative primer melting curve analysis software (LightCycler 480; Roche Diagnostics, Indianapolis, IN, USA). The mRNA expression levels of FGFR3, MMP9 and E-cadherin were normalized to those of GAPDH, and relative quantification was performed using the comparative cycle threshold method $\left(2-{ }^{\Delta \Delta \mathrm{Cq}}\right)(20)$. All PCR experiments were repeated $\geq 3$ times.

Western blot analysis. To analyze the protein expression levels of FGFR3, MMP9 and E-cadherin in A549 cells, transfected and untransfected cells were rinsed twice with precooled phosphate-buffered saline (Costar; Corning Life Sciences), prior to being homogenized in radioimmunoprecipitation assay (RIPA) buffer (Costar; Corning Life Sciences) supplemented with a protease and phosphatase inhibitor cocktail (Roche Diagnostics GmbH, Mannheim, Germany) at $36 \mathrm{~h}$ post-transfection. Cell lysates were centrifuged (GTR 21-1; Shanghai Medical Instruments Co., Ltd., Shanghai, China) at $14,000 \times \mathrm{g}$ for $10 \mathrm{~min}$ at $4^{\circ} \mathrm{C}$, and the supernatants were then mixed with $5 \mathrm{X}$ sodium dodecyl sulfate (SDS; Costar; Corning Life Sciences) loading sample buffer, boiled for $5 \mathrm{~min}$ and separated using $10 \%$ SDS-polyacrylamide gel electrophoresis at $60 \mathrm{~V}$ for $5 \mathrm{~h}$. Total cellular protein levels were quantified by Bradford assay (21) using standard protein solution and Coomassie BriliantBlue(R-250)(Generay BiotechCo.,Ltd.,Shanghai,China). Following electrophoresis, the proteins were transferred 
Table I. Effect of siRNA transfection on the invasion ability of A549 cells.

\begin{tabular}{|c|c|c|c|c|}
\hline Group & $\begin{array}{l}\text { No. of penetrated cells } \\
\text { /high-power field }\end{array}$ & $\begin{array}{l}\text { No. of transfected/ } \\
\text { blank cells }\end{array}$ & $\begin{array}{c}\text { Invasion inhibition } \\
\text { rate }(\%)\end{array}$ & P-value \\
\hline siRNA-855 & $32.49 \pm 8.25$ & 0.36 & 63.9 & 0.000004 \\
\hline siRNA-1447 & $34.68 \pm 11.48$ & 0.39 & 61.4 & 0.000006 \\
\hline siRNA-2076 & $34.29 \pm 9.44$ & 0.38 & 61.8 & 0.000005 \\
\hline NC-siRNA & $87.82 \pm 3.98$ & 0.97 & 2.0 & 0.745000 \\
\hline Blank & $89.95 \pm 0.73$ & - & - & - \\
\hline
\end{tabular}

Data are presented as the mean \pm standard deviation of 4 replicates. si, small interfering; NC, negative control.

to PVDF membranes (Shanghai Kehua Bio-Engineering Co., Ltd., Shanghai, China) by electrophoretic transfer. Membranes were blocked in $5 \%$ skimmed milk for $2 \mathrm{~h}$, rinsed with Tris-buffered saline containing Tween 20, and incubated overnight at $4^{\circ} \mathrm{C}$ with rabbit anti-human monoclonal FGFR3 (cat. no. ab137084; Abcam, Cambridge, MA, USA), MMP9 (cat. no. ab76003; Abcam) and E-cadherin (cat. no. 3195; Cell Signaling Technology, Inc., Danvers, MA, USA) primary antibodies (1:10,000 dilution in 5\% skimmed milk). Following 3 washes with Tris-buffered saline containing Tween 20, membranes were incubated with a secondary antibody [horseradish peroxidase-conjugated goat anti-rabbit immunoglobulin (Ig)G] (cat. no. CW0103M; Beijing Kang Century Biotechnology Co., Ltd., Beijing, China) for $12 \mathrm{~h}$ at $4^{\circ} \mathrm{C}$. Goat anti-mouse GAPDH (cat. no. CW0101M; Beijing Kang Century Biotechnology Co., Ltd.) was used as an internal standard. Signals were detected with a Novex ${ }^{\circledR}$ enhanced chemiluminescence (ECL) substrate reagent kit (Thermo Fisher Scientific, Inc.) for $1 \mathrm{~h}$ at $37^{\circ} \mathrm{C}$. For that purpose, developer A and B (obtained from the ECL Western Blotting kit; Costar; Corning Life Sciences) were mixed at a 1:1 ratio, prior to be added to the blots, which were subsequently exposed and imaged. The relative intensity of the bands was analyzed using ImageJ software (National Institutes of Health, Bethesda, MA, USA). All experiments were performed in triplicate.

Statistical analysis. Results were analyzed with SPSS statistical software version 18.0 (SPSS, Inc., Chicago, IL, USA). Data are represented as the mean \pm standard deviation of 5 replicates. Differences between multiple sets of data were compared by analysis of variance, while differences between 2 groups were compared by homogeneity of variance test. The significance level was considered to be $\alpha=0.05$, and $\mathrm{P}<0.05$ was considered to indicate a statistically significant difference.

\section{Results}

Morphology of A549 cells and transfection efficiency. Morphological changes in transfected A549 cells were observed under an inverted microscope at $6 \mathrm{~h}$ post-transfection. Prior to transfection, cells were adherent, fusiform and exhibited adequate growth, moderate size and a clear nucleolus, whereas $6 \mathrm{~h}$ following transfection cells were irregular, poorly adherent and exhibited shrinkage. Transfection efficiency was determined under an inverted fluorescence microscope (BX43; Olympus Corporation, Tokyo, Japan), based on the transfection efficiency exhibited by the FAM-siRNA-transfected cells (70-80\%; Fig. 1A and B).

siRNA-FGFR3 inhibits A549 invasion. To investigate the inhibitory effect of siRNA-855, siRNA-1447 and siRNA-2076 on the invasion of A549 cells, an invasion assay was performed with Matrigel upon inhibiting FGFR3 expression in A549 cells in vitro via siRNA-FGFR3 transfection. The results indicated that the inhibition of FGFR3 mediated by siRNA transfection led to a decrease in the invasion of siRNA-FGFR3-transfected cells (A-C groups), compared with the NC-siRNA and blank control groups $(\mathrm{P}<0.01$, Table I). These results suggest that siRNA-855, siRNA-1447 and siRNA-2076 are involved in the migration of A549 cells. In addition, siRNA-FGFR3 inhibited the invasion of A549 cells transfected with siRNA-855, siRNA-1447 and siRNA-2076, compared with the blank control group. Following transfection of A549 cells with siRNA for $36 \mathrm{~h}$, the results of the subsequent Transwell invasion assay demonstrated that the average number of invasive cells per high-power field in the siRNA-855, siRNA-1447 and siRNA-2076 groups was significantly reduced, compared with the blank control group (Fig. 1; magnification, x200). Thus, the number of invasive cells in the above groups was 0.36 , 0.39 and 0.38 times the number of invasive cells in the blank control group, respectively (Table I). Thus, the rate of inhibition of invasion for groups $\mathrm{A}, \mathrm{B}$ and $\mathrm{C}$ was determined to be 63.9, 61.4 and $61.8 \%(\mathrm{P}=0.000004, \mathrm{P}=0.000006$ and $\mathrm{P}=0.00005$, respectively; Table I). No significant differences were observed for the NC-siRNA group, where the number of invasive cells was determined to be 0.97 times that of the blank control group (P=0.745000; Fig. 1 and Table I).

mRNA expression of FGFR3, MMP9 and E-cadherin was altered in siRNA-transfected A549 cells. The Real-time PCR amplification efficiency was $100 \%$, the $2-\Delta \Delta \mathrm{Cq}$ method was applied to quantify fold-changes in the expression levels of FGFR3, MMP9 and E-cadherin in each cell group. As indicated in Tables II-IV, the number of mRNA corresponding to the FGFR3 gene was significantly reduced in the A, $\mathrm{B}$ and $\mathrm{C}$ groups, compared with the $\mathrm{E}$ group $(\mathrm{P}=0.000006$, $\mathrm{P}=0.000004$ and $\mathrm{P}=0.000004$, respectively). Compared with the E group, the mRNA expression levels of E-cadherin in the 
Table II. RNA interference inhibited the messenger RNA expression of fibroblast growth factor receptor 3 in A549 cells.

\begin{tabular}{lcccc}
\hline Group & siRNA-855 & siRNA-1447 & siRNA-2076 & NC-siRNA \\
\hline Fold-change & $0.22 \pm 0.89$ & $0.26 \pm 0.12$ & $0.26 \pm 0.09$ & $0.99 \pm 0.03$ \\
P-value & 0.000006 & 0.000004 & 0.000004 & 0.895000 \\
siRNA-855 & $32.49 \pm 8.25$ & 0.36 & 63.9 & 0.000004 \\
siRNA-1447 & $34.68 \pm 11.48$ & 0.39 & 61.4 & 0.000006 \\
siRNA-2076 & $34.29 \pm 9.44$ & 0.38 & 61.8 & 0.000005 \\
NC-siRNA & $87.82 \pm 3.98$ & 0.97 & 2.0 & 0.745000 \\
Blank & $89.95 \pm 0.73$ & 0.95 & 2.3 & 0.807000
\end{tabular}

Data are presented as the mean \pm standard deviation of 4 replicates; si, small interfering; NC, negative control.

Table III. RNA interference affected the messenger RNA expression of matrix metalloproteinase 9 in A549 cells.

\begin{tabular}{lcccc}
\hline Group & siRNA-855 & siRNA-1447 & siRNA-2076 & NC-siRNA \\
\hline Fold-change & $0.29 \pm 0.6$ & $0.35 \pm 0.55$ & $0.35 \pm 0.72$ & $0.94 \pm 0.09$ \\
P-value & 0.000007 & 0.000005 & 0.000004 & 0.761000 \\
siRNA-855 & $32.49 \pm 8.25$ & 0.36 & 63.9 & 0.000004 \\
siRNA-1447 & $34.68 \pm 11.48$ & 0.39 & 61.4 & 0.000006 \\
siRNA-2076 & $34.29 \pm 9.44$ & 0.38 & 61.8 & 0.000005 \\
NC-siRNA & $87.82 \pm 3.98$ & 0.97 & 2.0 & 0.745000 \\
Blank & $89.95 \pm 0.73$ & 0.95 & 2.3 & 0.807000
\end{tabular}

Data are presented as the mean \pm standard deviation of 4 replicates; si, small interfering; NC, negative control.

Table IV. RNA interference affected the messenger RNA expression of E-cadherin in A549 tells.

\begin{tabular}{lcccc}
\hline Group & siRNA-855 & siRNA-1447 & siRNA-2076 & NC-siRNA \\
\hline Fold-change & $2.35 \pm 0.58$ & $2.13 \pm 0.32$ & $2.12 \pm 0.20$ & $1.06 \pm 0.15$ \\
P-value & 0.000005 & 0.000003 & 0.000003 & 0.803000 \\
siRNA-855 & $32.49 \pm 8.25$ & 0.36 & 63.9 & 0.000004 \\
siRNA-1447 & $34.68 \pm 11.48$ & 0.39 & 61.4 & 0.000006 \\
siRNA-2076 & $34.29 \pm 9.44$ & 0.38 & 61.8 & 0.000005 \\
NC-siRNA & $87.82 \pm 3.98$ & 0.97 & 2.0 & 0.745000 \\
Blank & $89.95 \pm 0.73$ & 0.95 & 2.3 & 0.807000 \\
\hline
\end{tabular}

Data are presented as the mean \pm standard deviation of 4 replicates; si, small interfering; NC, negative control.

siRNA-transfected groups increased significantly $(\mathrm{P}=0.000005$, $\mathrm{P}=0.000003$ and $\mathrm{P}=0.000003$ for groups $\mathrm{A}, \mathrm{B}$ and $\mathrm{C}$, respectively), while the mRNA expression levels of MMP9 in these groups were significantly reduced $(\mathrm{P}=0.000007, \mathrm{P}=0.000005$ and $\mathrm{P}=0.000004$ for groups $\mathrm{A}, \mathrm{B}$ and $\mathrm{C}$, respectively). Compared with the blank control group, the cDNA copy number of FGFR3, MMP9 and E-cadherin in the NC-siRNA group did not change significantly ( $\mathrm{P}>0.05$; Tables II-IV). These results indicated that siRNA transfection with Lipo2000 markedly inhibited the mRNA expression of FGFR3 in a specific manner, which led to a marked reduction in the mRNA expression levels of MMP9 and a marked increase in the mRNA expression levels of E-cadherin in siRNA-FGFR3-transfected A549 cells.
Protein expression of FGFR3, MMP9 and E-cadherin was altered in siRNA-transfected A549 cells. Western blot analysis demonstrated that, compared with groups D and E, the protein levels of FGFR3 and MMP9 were downregulated in A-C groups, while the protein levels of E-cadherin were upregulated in these groups, according to the lower and higher gray, respectively, of the protein bands in the A-C groups (Fig. $1 \mathrm{H})$. Image J software was used to analyze the gray values of the different protein bands. Compared with the E group, the FGFR3/GAPDH and MMP9/GAPDH ratios in the A-C groups were significantly decreased, while the E-cadeherin/GAPDH ratio in these groups was significantly increased, compared with the E group $(\mathrm{P}<0.01$; Tables V-VII). No significant differences 
Table V. Ratio of FGFR3/GAPDH protein expression in each group.

\begin{tabular}{lccccc}
\hline Group & siRNA-855 & siRNA-1447 & siRNA-2076 & NC-siRNA & Blank \\
\hline FGFR3/GAPDH & $0.200 \pm 0.026$ & $0.190 \pm 0.020$ & $0.187 \pm 0.038$ & $0.553 \pm 0.087$ & $0.620 \pm 0.0095$ \\
P-value & 0.000009 & 0.000007 & 0.000006 & 0.218000 & 0.541000 \\
siRNA-855 & $32.49 \pm 8.25$ & 0.36 & 63.9 & 0.000004 & - \\
siRNA-1447 & $34.68 \pm 11.48$ & 0.39 & 61.4 & 0.000006 \\
siRNA-2076 & $34.29 \pm 9.44$ & 0.38 & 61.8 & 0.000005 & - \\
NC-siRNA & $87.82 \pm 3.98$ & 0.97 & 2.0 & 0.745000 & - \\
Blank & $89.95 \pm 0.73$ & - & - & -
\end{tabular}

Data are presented as the mean \pm standard deviation of 4 replicates; si, small interfering; NC, negative control; FGFR3, fibroblast growth factor receptor 3; GAPDH, glyceraldehyde-3-phosphate dehydrogenase.

Table VI. Ratio of E-cadherin/GAPDH protein expression in each group.

\begin{tabular}{lccccc}
\hline Group & siRNA-855 & siRNA-1447 & siRNA-2076 & NC-siRNA & Blank \\
\hline E-cadherin/GAPDH & $0.627 \pm 0.074$ & $0.743 \pm 0.057$ & $0.647 \pm 0.083$ & $0.257 \pm 0.055$ & $0.267 \pm 0.078$ \\
P-value & 0.000092 & 0.000008 & 0.000059 & 0.865000 & - \\
\hline
\end{tabular}

Data are presented as themean \pm standard deviation of 4 replicates; si, smallinterfering; NC, negativecontrol.GAPDH,glyceraldehyde-3-phosphate dehydrogenase.

Table VII. Ratio of MMP9/GAPDH protein expression in each group.

\begin{tabular}{lccccc}
\hline Group & siRNA-855 & siRNA-1447 & siRNA-2076 & NC-siRNA & Blank \\
\hline MMP9/GAPDH & $0.363 \pm 0.055$ & $0.357 \pm 0.080$ & $0.360 \pm 0.134$ & $0.770 \pm 0.120$ & $0.750 \pm 0.066$ \\
P-value & 0.000600 & 0.001000 & 0.001000 & 0.804000 & - \\
\hline
\end{tabular}

Data are presented as the mean \pm standard deviation of 4 replicates; si, small interfering; NC, negative control. MMP9, matrix metalloproteinase 9; GAPDH, glyceraldehyde-3-phosphate dehydrogenase.

in FGFR3, MMP-9 and E-cadherin expression were observed between the A-C groups or between groups $\mathrm{D}$ and $\mathrm{E}(\mathrm{P}>0.01$; Tables V-VII). These results suggest that the protein expression of FGFR3, encoded by FGFR3 mRNA, was inhibited, possibly due to the specific degradation of FGFR 3 mRNA induced by siRNA-FGFR3, which led to a decrease in the protein expression levels of MMP9 and an increase in the protein expression levels of E-cadherin.

\section{Discussion}

The process of carcinoma metastasis consists of a series of sequential steps (4). Initially, tumor cells are detached from the ECM, and the surrounding tissue is then invaded (4). Losing cell-cell adhesion and gaining motility are important for carcinoma cells to separate from neighboring cells and invade other tissues (4). EMT is a multistage process involving major alterations in cell morphology, cell-cell and cell-matrix adhesions. ETM is important for the motile and invasive capabilities of cells, which are essential in malignant tumor progression and metastasis (14). A hallmark of EMT is the loss of expression of
E-cadherin, which is a key cell-cell adhesion molecule (22). It has been demonstrated that the ability of invasion and metastasis of certain invasive carcinoma cells may be altered by forcing the expression of E-cadherin (22-24).

ECM dysregulation and remodeling are essential for the progression of neoplastic processes, and MMP-mediated ECM degradation has been demonstrated to lead to cancer cell invasion and metastasis (25). Previous studies on stromal cells undergoing EMT have revealed that EMT may be the most critical and abnormal signaling pathway that occurs during development and metastasis of lung cancer (22). MMP9 has been identified as a critical component of the multifunctional MMP family of enzymes, which act as ECM-degrading proteases and may promote metastasis $(26,27)$. The hypothetical role of MMP9 as a metastasis promoter is supported by a previous study (28).

The FGFR3 gene is located in the 4p16.3 region of the chromosome, which consists of 19 exons and 18 introns expanding across $16.5 \mathrm{~kb}$ and encodes a 4.4-kb mRNA molecule (29-31). FGFR 3 is a member of the FGFR family of tyrosine kinase receptors, and consists of an extracellular domain that includes a 
signal peptide, followed by 3 Ig-like domains, an acidic box, a transmembrane domain and an intracellular tyrosine kinase domain (32). FGFR3 is activated by binding of the FGF ligand to the extracellular Ig-like domains II and III (3). Subsequently, the trans-autophosphorylation of tyrosine residues in the cytoplasmic domain of FGFR3 stimulates the intrinsic catalytic activity of the receptor, and leads to the activation of downstream signaling pathways $(13,33)$. FGFR3 has been demonstrated to be involved in the RAS/RAF/MEK/MAPK signaling pathway through the activation of $\mathrm{p} 90$ ribosomal S6 kinase $(13,33)$.

Previous studies have reported that FGFR3 is overexpressed in lung cancer and bone metastasis of lung adenocarcinoma; thus, FGFR3 may be regarded as a potential target for cancer therapy $(3,10,11)$. However, the effect of silencing FGFR3 on the inhibition of invasion of lung carcinoma remains unknown. Therefore, the present study aimed to inhibit the expression of FGFR3 in lung cancer cells in order to evaluate its effects on the invasion ability of NSCLC. For that purpose, the association between FGFR3, E-cadherin and MMP9 expression in A549 cells was investigated by siRNA targeting FGFR3. In addition, the mRNA and protein expression levels of FGFR3, E-cadherin and MMP9 were analyzed in siRNA-FGFR3-transfected and control groups.

Previous in vivo and in vitro studies have demonstrated that the upregulation of FGFR3 increased cancer cell invasion and metastases in breast (12) and lung cancer (10). A hallmark of cancer is its self-sufficiency in terms of growth signals, which is frequently driven by RTK-dependent growth factor signaling pathways (34). These pathways are important during the development and adult life of multicellular organisms $(2,3)$. FGFR3 belongs to the RTK family, and FGF/FGFR3 signaling has been frequently observed to occur in certain NSCLC cell lines via the Ras/RAF/MEK/MAPK signaling pathway, which regulates key cellular processes such as cell proliferation, migration and survival $(25,35)$. A previous study has reported that FGFR signaling is active and important in cells undergoing EMT, which is induced by transforming growth factor- $\beta$ (14). EMT is involved in the progression and metastasis of cancer (14). In the present study, marked upregulation of E-cadherin and downregulation of MMP9 via siRNA targeting FGFR3 was observed in lung cancer A549 cells. Furthermore, the results of Transwell assay demonstrated that the invasion ability of siRNA-FGFR3-transfected cells significantly decreased, compared with control cells. Taken together, these results indicate that FGFR3 is associated with tumor invasion and may promote the expression of E-cadherin and MMP9, which are associated with EMT and ECM remodeling, respectively, in lung cancer. However, the direct mechanism between FGFR3 and MMPs remains to be elucidated. Therefore, siRNA targeting FGFR3 may act as a tumor suppressor through the regulation of FGFR3. Thus, RNAi technology may serve as a potential target of NSCLC therapy.

In conclusion, the present study suggests that downregulation of FGFR3 via siRNA-FGFR3 in NSCLC may lead to a decrease in tumor cell invasion. In addition, the expression of FGFR3 was observed to be significantly correlated with the expression of MMP9 and E-cadherin in the present study.
According to these results, FGFR3 may be an important marker of NSCLC metastasis and a potential therapeutic target. Further studies are required to clarify the mechanisms of FGFR3 in EMT and ECM remodeling in NSCLC.

\section{Acknowledgements}

The present study was supported by the Natural Science Foundation of Shandong Province (Jinan, China; grant no. W2012FZ078) and Wu Jieping Medical Foundation (Beijing, China; grant no. 320.6750.13210).

\section{References}

1. Siegel R, Ward E, Brawley O and Jemal A: Cancer statistics, 2011: The impact of eliminating socioeconomic and racial disparities on premature cancer deaths. CA Cancer J Clin 61: 212-236, 2011

2. Siegel R, DeSantis C, Virgo K, Stein K, Mariotto A, Smith T, Cooper D, Gansler T, Lerro C, Fedewa S, et al: Cancer treatment and survivorship statistics, 2012. CA Cancer J Clin 62: 220-241, 2012.

3. Dat T, Matsuo T, Yoshimaru T, Kakiuchi S, Goto H, Hanibuchi M, Kuramoto T, Nishioka Y, Sone S and Katagiri T: Identification of genes potentially involved in bone metastasis by genome-wide gene expression profile analysis of non-small cell lung cancer in mice. Int J Oncol 40: 1455-1469, 2012.

4. Langley RR and Fidler IJ: The seed and soil hypothesis revisited - the role of tumor-stroma interactions in metastasis to different organs. Int J Cancer 128: 2527-2535, 2011.

5. Larsen JE and Minna JD: Molecular biology of lung cancer: Clinical implications. Clin Chest Med 32: 703-740, 2011.

6. Miele E, Spinelli GP, Miele E, Di Fabrizio E, Ferretti E, Tomao S and Gulino A: Nanoparticle-based delivery of small interfering RNA: Challenges for cancer therapy. Int J Nanomedicine 7: 3637-3657, 2012.

7. Joo MK, Yhee JY, Kim SH and Kim K: The potential and advances in RNAi therapy: Chemical and structural modifications of siRNA molecules and use of biocompatible nanocarriers. J Control Release 193: 113-121, 2014.

8. Chen Y, Gu H, Zhang DS, Li F, Liu T and Xia W: Highly effective inhibition of lung cancer growth and metastasis by systemic delivery of siRNA via multimodal mesoporous silica-based nanocarrier. Biomaterials 35: 10058-10069, 2014.

9. Shi L, Zhang B, Sun X, Lu S, Liu Z, Liu Y, Li H, Wang L, Wang X and Zhao C: MiR-204 inhibits human NSCLC metastasis through suppression of NUAK1. Br J Cancer 111: 2316-2327, 2014.

10. Kang J, Lee SY, Lee SY, Kim YJ, Park JY, Kwon SJ, Na MJ, Lee EJ, Jeon HS and Son JW: MicroRNA-99b acts as a tumor suppressor in non-small cell lung cancer by directly targeting fibroblast growth factor receptor 3. Exp Ther Med 3: 149-153, 2012.

11. Lamont FR, Tomlinson DC, Cooper PA, Shnyder SD, Chester JD and Knowles MA: Small molecule FGF receptor inhibitors block FGFR-dependent urothelial carcinoma growth in vitro and in vivo. Br J Cancer 104: 75-82, 2011.

12. Smid M, Wang Y, Klijn JG, Sieuwerts AM, Zhang Y, Atkins D, Martens JW and Foekens JA: Genes associated with breast cancer metastatic to bone. J Clin Oncol 24: 2261-2267, 2006.

13. Kang S, Dong S, Gu TL, Guo A, Cohen MS, Lonial S, Khoury HJ, Fabbro D, Gilliland DG, Bergsagel PL, et al: FGFR3 activates RSK2 to mediate hematopoietic transformation through tyrosine phosphorylation of RSK2 and activation of the MEK/ERK pathway. Cancer Cell 12: 201-214, 2007.

14. Wang X, Lu H, Urvalek AM, Li T, Yu L, Lamar J, DiPersio CM, Feustel PJ and Zhao J: KLF8 promotes human breast cancer cell invasion and metastasis by transcriptional activation of MMP9. Oncogene 30: 1901-1911, 2011.

15. Tu CL and You M: Obligatory roles of filamin A in E-cadherin-mediated cell-cell adhesion in epidermal keratinocytes. J Dermatol Sci 73: 142-151, 2014.

16. Jiang XB, Wang JS, Liu DH, Yuan WS and Shi ZS: Overexpression of matrix metalloproteinase- 9 is correlated with carotid intraplaque hemorrhage in a swine model. J Neurointerv Surg 5: 473-477, 2013. 
17. Stenvold H, Donnem T, Andersen S, Al-Saad S, Al-Shibli K, Busund LT and Bremnes RM: Overexpression of matrix metalloproteinase-7 and -9 in NSCLC tumor and stromal cells: Correlation with a favorable clinical outcome. Lung Cancer 75: 235-241, 2012.

18. Shuman Moss LA, Jensen-Taubman S and Stetler-Stevenson WG: Matrix metalloproteinases: Changing roles in tumor progression and metastasis. Am J Pathol 181: 1895-1899, 2012.

19. Zhang B, Xie C, Zhong J, Chen H, Zhang H and Wang X: A549 cell proliferation inhibited by RNAi mediated silencing of the Nrf2 gene. Biomed Mater Eng 24: 3905-3916, 2014.

20. Schmittgen TD and Livak KJ: Analyzing real-time PCR data by the comparative C(T) method. Nat Protoc 3: 1101-1108, 2008.

21. Ong KJ, MacCormack TJ, Clark RJ, Ede JD, Ortega VA, Felix LC, Dang MK, Ma G, Fenniri H, Veinot JG and Goss GG: Widespread nanoparticle-assay interference: implications for nanotoxicity testing. PLoS One 9: e90650, 2014:

22. Nijkamp MM, Span PN, Hoogsteen IJ, van der Kogel AJ, Kaanders JH and Bussink J: Expression of E-cadherin and vimentin correlates with metastasis formation in head and neck squamous cell carcinoma patients. Radiother Oncol 99: 344-348, 2011.

23. Chen ZL, Zhao XH, Wang JW, Li BZ, Wang Z, Sun J, Tan FW, Ding DP, Xu XH, Zhou F, et al: MicroRNA-92a promotes lymph node metastasis of human esophageal squamous cell carcinoma via E-cadherin. J Biol Chem 286: 10725-10734, 2011.

24. Liu Q, Wang YD, Jing SH, Wang XL, Cheng YJ and Wu FP Expression of E-cadherin in nasopharyngeal carcinoma and its relationship with cervical lymph node metastasis. Zhonghua Zhong Liu Za Zhi 32: 425-428, 2010 (In Chinese).

25. Kessenbrock K, Plaks V and Werb Z: Matrix metalloproteinases: Regulators of the tumor microenvironment. Cell 141: 52-67, 2010.

26. Reeves CV, Wang X, Charles-Horvath PC, Vink JY, Borisenko VY, Young JA and Kitajewski JK: Anthrax toxin receptor 2 functions in ECM homeostasis of the murine reproductive tract and promotes MMP activity. PLoS One 7: e34862, 2012.
27. de Oliveira Demarchi AC, Zambuzzi WF, Paiva KB, da Silva-Valenzuela M, Nunes FD, de Cássia Sávio Figueira R, Sasahara RM, Demasi MA, Winnischofer SM, Sogayar MC and Granjeiro JM: Development of secondary palate requires strict regulation of ECM remodeling: Sequential distribution of RECK, MMP-2, MMP-3, and MMP-9. Cell Tissue Res 340: 61-69, 2010.

28. Liu J, Ping W, Zu Y and Sun W: Correlations of lysyl oxidase with MMP2/MMP9 expression and its prognostic value in non-small cell lung cancer. Int J Clin Exp Pathol 7: 6040-6047, 2014.

29. Liao RG, Jung J, Tchaicha J, Wilkerson MD, Sivachenko A, Beauchamp EM, Liu Q, Pugh TJ, Pedamallu CS, Hayes DN, et al: Inhibitor-sensitive FGFR2 and FGFR3 mutations in lung squamous cell carcinoma. Cancer Res 73: 5195-5205, 2013.

30. Zhang Q, Jiang HO, Quan QL, Li J, He T and Huang XS: Mutation analysis of FGFR3 gene in a family featuring hereditary dwarfism. Zhonghua Yi Xue Yi Chuan Xue Za Zhi 28: 705-707, 2011 (In Chinese).

31. Sahlin P, Tarnow P, Martinsson T and Stenman G: Germline mutation in the FGFR3 gene in a TWIST1-negative family with Saethre-Chotzen syndrome and breast cancer. Genes Chromosomes Cancer 48: 285-288, 2009.

32. Haugsten EM, Wiedlocha A, Olsnes S and Wesche J: Roles of fibroblast growth factor receptors in carcinogenesis. Mol Cancer Res 8: 1439-1452, 2010.

33. Blick C, Ramachandran A, Wigfield S, McCormick R, Jubb A, Buffa FM, Turley H, Knowles MA, Cranston D, Catto J and Harris AL: Hypoxia regulates FGFR3 expression via HIF-1 $\alpha$ and miR-100 and contributes to cell survival in non-muscle invasive bladder cancer. Br J Cancer 109: 50-59, 2013.

34. Hanahan D and Weinberg RA: The hallmarks of cancer. Cell 100: 57-70, 2000.

35. Schlessinger J: Cell signaling by receptor tyrosine kinases. Cell 103: 211-225, 2000 